\title{
The Psychological Features of Personality Protection under Conditions of Stress Caused by Forced Isolation
}

\author{
Guzel S. Gabdreeva ${ }^{1}$ \\ ${ }^{1}$ Kazan (Volga) Federal University, Kazan, Russia \\ Correspondence: Guzel S. Gabdreeva, Kazan (Volga) Federal University, Kremlevskaya Street 18, Kazan, \\ Tatarstan, 420008, Russia.
}

Received: October 24, 2014

Accepted: December 3, 2014 Online Published: December 18, 2014

doi:10.5539/res.v7n1p101

URL: http://dx.doi.org/10.5539/res.v7n1p101

\begin{abstract}
The aim of this article is to present a series of studies on coping with traumatic events in three different settings and groups. The leading approach to research the tendency toward pseudocompensation was more strongly expressed among women, which corresponds to previous data showing that women have a lower stress tolerance than men. The first group consisted of convicts serving sentences in special facilities, the second of chronically ill people, and the third of people experiencing chronic stress due to "conditional isolation". The control group consisted of psychologically healthy people. The results of this article presents suggest that adaptedness is an integral feature of personality. The content of this article can be used in psychological rehabilitation to apply a differentiated approach to those experiencing chronic stress associated with the partial or complete loss of freedom.
\end{abstract}

Keywords: pseudo compensation, self-pity complex, fear-complex, isolation-related stressors, correlation analysis

\section{Introduction}

The problem of stress and stress disorders is one of the most pressing in contemporary psychology. During the second half of the twentieth century a current of psychology emerged that studied "coping mechanisms" in traumatic situations that has produced a large number of empirical and theoretical studies. This problem has found expression in the psychological literature under the concepts of "coping with stress" and "coping behavior."

The first concept (coping with stress) refers to an effort aimed at the prevention, elimination, or reduction of the effect of stressors and the second (coping behavior) describes the means used to bring a person out of a stressful situation and is viewed from the perspective of two main functions: regulating the person's state and managing the problems that cause distress (Bodrov, 2006; Posokhova, 2001). Coping that is aimed at a person's state is determined by the cognitive, emotional, and behavioral features of the process of reducing mental tension (emotionally focused coping). Efforts to eliminate the effect of the stressor are viewed as active coping behavior aimed at solving a problem. Problem-focused coping is conscious behavior carried out with the goal of changing or transforming a situation susceptible to control or adapting when control is not possible. Passive coping behavior is oriented toward intrapsychic means for overcoming stress that are defense mechanisms designed to deal with growing tension. Emotional balance is, of course, more often restored using passive strategies (not by solving the problem) when someone lacks the knowledge, ability, or real option of reducing pressure from the stressor. Coping behavior is achieved through coping resources (personal adaptive reserves that permit optimal adaptation to stressful situations) and coping strategies (ways to overcome stress).

L. I. Antsyferova makes the point that the correct choice of a strategy to cope with traumatic events depends on the ability to cognitively assess a difficult situation. The main result of this cognitive assessment is a determination as to whether the situation can be controlled or is impossible to change. If people feel that they can control the situation, they apply a constructive strategy to transform it (Antsyferova, 1994). The balanced use of active coping strategies aimed at solving a problem and appropriate to the situation is considered an indicator of a high level of adaptability.

When objective circumstances render it impossible to alter stress situations and solve the problems associated 
with them, coping behavior is oriented toward changing the mental state through the unconscious use of psychological defenses. Defense processes are aimed at preserving people's integrity and independence, their self-esteem and self-respect. The main task of defense mechanisms is to control and suppress negative mental states that arise under the influence of a stressor when the stressor itself is still in effect. R. M. Granovskaia believes that, by neutralizing signals that are dangerous for the inner world, this defense serves as a stabilization system that manifests itself either in eliminating negative emotions, or at least minimizing them (Granovskaia, 2003). A mature system of psychological defense comes in the form of an individual repertoire of defense mechanisms and defense behaviors, according to Bogomolov and Portnova (2005), while Kruzhkova (2006) clarifies that certain people prefer certain defenses that become an integral part of their individual approach to overcoming difficulty.

In 1936 Anna Freud identified nine types of defenses, including regression, repression, and projection. Ten years later she added two more items to the list. Sigmund Freud used four others: denial, rationalization, displacement, derealization. By now, a multitude of other defenses have been described. Despite some disagreement over just what should be included on the "list," all authors agree that all defenses can be classified using criteria of primitive-ness or maturity.

When they feel helpless in the face of traumatic reality, insufficiently adapted people resort, as a rule, to immature, primitive defense mechanisms. In situations of chronic stress that are perceived as inescapable, the constant use of primitive psychological defenses becomes entrenched in the form of complexes, and individual mental phenomena become integrated into a structural whole that determines the features of behavior. The complex of symptoms that took shape to permit the personality to remain intact in the face of destabilizing emotional experiences and that achieved more or less successful adaptations loses relevance when the situation that prompted the symptoms to develop changes. However, since they are persistent personality formations they continue to exist under new conditions, despite the fact that they have objectively outlived their usefulness. No longer appropriate to the situation, the personality neoformations that served as adaptations to past conditions become ineffectual and at times play a negative role in situations that demand readaptation. In light of this, research into the ways in which compensatory complexes manifest themselves depending on the conditions of the stress situation is timely. A number of authors researching the problems of stress believe that isolation, even partial, is a significant stressor (Gabdreeva, 2002; Mulleneisen, 1993, etc). Since human behavior is socially conditioned and largely regulated, there is particular interest in situations involving the phenomenon of complete or partial social isolation.

\section{Materials and Methods}

The isolation-related stressors that we investigated were associated with limitations on freedom and the negative situations, perceived as inescapable, stemming from them: the conditions of forced, long-term stays in the facilities of the correctional system and the consequences of serious chronic diseases that lead to disability and limitations in freedom of movement. Along with these stress situations, we also focused on the situations confronting immigrants living in our country based on long-term labor contracts. In the first case, we studied people subjected to chronic stress caused by incarceration (convicts serving sentences in special facilities- 58 men and 63 women). The second study sample included the chronically ill whose freedom was limited by serious disorders occurring in adulthood and leading to disability (severe orthopedic disorders confining them to wheelchairs or loss of sight or hearing-18 men and 20 women). The third group of subjects, who were experiencing chronic stress due to "conditional isolation" (Miullenaizen, 1993), included immigrants living outside their native countries in the former Soviet Union and beyond for extended periods (29 men and 27 women). The control group consisted of psychologically healthy people of the same age who were not currently experiencing long-term and inescapable chronic stress associated wilh fort id isolation of any kind-bookkeepers, salespeople, drivers, health-care workers, office employees - 37 people (17 men and 20 women). In total, 245 people were studied -115 men and 130 women.

Comparative analysis of the data identified a number of patterns in the use of primitive psychological defenses - "pseudocompensation"- by people living in situations of chronic stress. It turned out that regardless of the specific nature of the stressor, people living under the pressure of difficult situations typically applied essentially all the primitive defense complexes identified by the author using our diagnostic method. Furthermore, the tendency toward pseudocompensation was more strongly expressed among women, which corresponds to previous data showing that women have a lower stress tolerance than men (Gabdreeva, 2002).

\section{Results}

It was discovered that in the control group the numerical value for defense complexes was much lower than in 
the inmate, disabled, and immigrant groups. For example, the arithmetic mean value for all indicators of pseudocompensation within the group of male convicts was 3.98 while for women it was 4.42 (above the mean), and within the disabled group it was 3.67 for men and 4.03 for women. Among immigrants the figures were 2.91 for men and 3.27 for women. Within the control group, the mean figure for men was 0.67 (a low level) and for women from this group it was much higher-1.35, but in both cases the indicator was lower than the mean. Furthermore, in the control group, the complexes of "withdrawal into disease," "impairment," "fear," "foreboding," and "gullibility" were essentially absent among men (for women they were minimal). The female control group did not manifest "tyrant" or "guilt" complexes. The only complexes expressed in the male control group (at the level of the mean value) were "laziness" and "feigned ingenuousness." These same complexes, plus the "self-promotion," "devious-ness," and "fading youth" complexes, were found in the female group. The remaining complexes included in Table 1 have minimal mean values because they were exhibited by only a small number of subjects.

The group with the highest indicators for pseudocompensation were inmates, both men and women. Furthermore, the male sample had the highest indicators for "tyrant," "guilt," "fading youth," and "deviousness" complexes. For the female sample the highest indicators were for "guilt," "self-pity," "fear," "foreboding," "withdrawal into disease," and "gullibility."

People with sight, hearing, and mobility disabilities exhibited the same high indicators for pseudocompensation as the inmate group. Among handicapped males, the most strongly expressed complexes were "guilt," "impairment," and "demonstrative weakness." Next in intensity were "withdrawal into disease," "foreboding," and "self-pity" complexes. The values for remaining complexes were above the mean. Disabled females manifested even higher pseudocompensation values than males. The most strongly expressed complexes among these women were "self-pity," "withdrawal into disease," and "demonstrative weakness," followed by "impairment," "guilt," and "fear." All other complexes had above-mean values, as was the case for the male sample.

In the immigrant sample, as noted above, the numerical values for indicators of psychological defenses were lower than for the inmate or disabled samples. Nevertheless they were more strongly expressed than in the control group. Indicators for types of pseudo compensation such as "envy," "fear," and "greed" complexes were above average in men while the corresponding complexes for women were "khaliavshchik" (khaliava is a Russian slang expression for getting something without any particular effort-"freebies"), "foreboding," and "impairment." The values for remaining types of pseudo compensation were also above average or high on the diagnostic scale.

The results attest to the fact that people in difficult life situations that provoke the development of chronic stress actively use primitive psychological defenses. Furthermore, regardless of the source of the isolation-related stressor - whether incarceration, disability, or long-term residence in a foreign country — commonalities can be identified in the application of pseudo compensation (see Table 1).

Table 1. Tendencies to use pseudo compensation

\begin{tabular}{lllllllll}
\hline & \multicolumn{2}{c}{ Control group } & \multicolumn{2}{c}{ Inmates } & \multicolumn{2}{c}{ Disabled } & \multicolumn{2}{c}{ Immigrants } \\
Level & Female & Male & Female & Male & Female & Male & Female & Male \\
\hline $\mathbf{1}$ & CSPr & CLaz & CGlt & CTyr & CSPt & CGlt & CKhal & CEnv \\
$\mathbf{2}$ & CDev & CFI & CSPt & CGlt & CWD & CImp & CFb & CFr \\
$\mathbf{3}$ & CLaz & CSPr & CFr & CFY & CDW & CDW & CImp & CGr \\
$\mathbf{4 4}$ & CFY & CSup & CFb & CDev & CImp & CWD & CEnv & CKhal \\
$\mathbf{5 5}$ & CKhal & CGr & CWD & CFY & CGlt & CFb & CSPt & CImp \\
$\mathbf{6 6}$ & CEnv & CEnv & CGul & CSPt & CFr & CSPt & CFr & CLaz \\
$\mathbf{7 7}$ & CGul & CKhal & CKhal & CFb & CFY & CGul & CDW & CSPt \\
$\mathbf{8 8}$ & CDW & CGul & CImp & CGul & CGul & CEnv & CDev & CFr \\
$\mathbf{9 9}$ & CSup & CFY & CDev & CImp & CEnv & CFr & CGlt & CGlt \\
$\mathbf{1 1 0}$ & CGr & CDW & CDW & CFr & CFI & CLaz & CLaz & CDev
\end{tabular}

Notes. CWD—withdrawal into disease complex; CDW—demonstrative weakness complex; CLaz—laziness 
complex; CDev-deviousness complex; CTyr-turant complex; CSup-superiority complex; CSPr—self-promotion complex; CSPt—self-pity complex; CFY_fading youth complex; CFI—feigned ingenuousness; CEnv-envy complex; CGr-greed complex; CGlt-guilt complex; CImp-impairment complex; $\mathrm{CFr}$-fear complex; $\mathrm{CFb}$-foreboding complex; CKhal—Khaliava complex; CGul—gullibility complex.

The first thing one notices is the prominence of the guilt complex. Among male respondents, the indicator for this complex was highest for the disabled. Compared to other subjects, men who became disabled experienced a sense of guilt toward their family and loved ones because they were not able to fulfill the hopes that were placed on them and to repay family members for all their kindness. Among male inmates, this complex is also present, although it occupies second place behind the tyrant complex. Among female respondents the guilt complex takes first place among inmates. Male and female immigrants experience approximately the same degree of guilt for the decision that greatly changed their chosen path in life. However, in the control group the guilt complex is not prominent among other complexes. It occasionally appears among some males and is almost entirely undetected in women.

Members of the control group essentially do not exhibit self-pity, but this complex is strongly reflected among disabled females, for whom it occupies first place. Among female inmates this complex occupies second place after a sense of guilt, and among female immigrants it occupies fifth place in the hierarchy. Among men, both the disabled and inmates, the self-pity complex occupies sixth place, while for immigrant men it is in seventh place.

Pseudo compensation in the form of the withdrawal into disease complex occupies second place among disabled females. However the hope of early release due to illness is nurtured even by perfectly healthy female inmates, among whom this complex is in fifth place. Among male respondents, withdrawal into disease is to a certain extent present for the disabled and is not manifested among male inmates. This complex is largely absent among immigrants and those in the control group.

The research into psychological defenses confirms the idea that all types of isolation are associated with a specific sense of fear (Miullenaizen, 1993). The fear complex is present to some extent throughout the study sample of individuals living with chronic isolation-related stress. Among men, it is most present in the immigrant group, for which it occupies second place. Among female criminals serving sentences, this indicator occupies third place. Among disabled females and female inmates this complex occupies ninth and tenth place respectively.

Everyone living in difficult life situations, without exception, to some extent experiences the impairment complex, which was essentially absent among representatives of the control group.

The data support the view that the main thing that provokes psychological defenses is a difficult situation perceived as inescapable. However, no less important is another determinant of psychological defenses: personality. This is attested to by the results of an analysis we performed of the interconnections between indicators of the propensity to use pseudo compensation and indicators of mental potential, the spiritual potential of a person's semantic-life orientation, personality traits, character accentuation, temperament, and physical health. The "target" was chosen for psychodiagnostic reasons on the basis of existing ideas about individual psychological features as determinants of psychological personality adaptation under difficult life circumstances, features that form the basis for a person's adaptive resources (Bodrov, 2006; Kruzhkova, 2006; Maklakov, 2001).

The results of the correlation analysis suggest that the inclination to use primitive psychological defenses (specifically pseudo compensation) is incorporated into the structure of people's adaptive complex when they find themselves under chronic isolation-related stress. An analysis was performed of interconnections within the correlative structure incorporating, on the one hand, indicators of individual psychological properties enabling adaptive resources, and, on the other, indicators of an inclination toward pseudo compensation. It turned out that all diagnosed properties were closely interrelated $(\mathrm{p}<0.05$ and $\mathrm{p}<0.01)$ only in groups consisting of individuals living under conditions that promote the development of chronic stress: incarcerated convicts; disabled people who had partially or completely lost their sight, hearing, or mobility; and immigrants.

In the structure of indicators for the convict sample-individuals with the highest degree of isolation due to incarceration-within the male group there were seventeen interconnections between compensation indicators and other properties; in the female group there were eleven. If we consider that the diagnostic method used by the author in our study identified a total of eighteen indicators of an inclination toward psychological defense (in the form of pseudo compensation) then it can be claimed that the data concerning the number of interconnections 
attest to an active interaction between psychological defensive complexes and other studied properties. The accentuation of character (hyperthymic accentuation in particular) and properties of temperament (plasticity, hardiness, quickness) are determinants of an inclination to use pseudocompensation among female convicts, in that these are the very indicators with which the diagnosed complexes are most closely associated $(p<0.001)$. In the male inmate sample there was a much broader range of personality determinants: in addition to those found in the female group, there was also the degree of controllability of life and certain personality traits such as shyness, anxiety, and poor self-control.

The greatest number of interconnections with indicators for individual psychological traits was with the guilt complex. This complex was experienced by female inmates distinguished by low values for the "reserve-expressiveness" personality factor $(\mathrm{p}<0.05)$. In other words, restrained, cautious, prudent, quiet women, inclined to exaggeration and pessimistic in their perception of reality are more likely to experience the guilt complex. Expressive women, characterized by impulsivity, expansiveness, and a carefree attitude use this psychological defense much more rarely. A high level of anxiety in women is compensated for through the development of the tyrant complex (an interconnection was found where $\mathrm{p}<0.01$ ) manifested from an aggressive-defensive position: "I am capable of being cruel and demanding of people," "Someday a lot of people will regret that they did not give me my due," and so on. There is also a strong correlation between severe anxiety and indicators for the superiority complex, manifested by assertions such as, "A lot of people envy me because I'm better than they are," "I'll achieve more than others," and so on. Certain features of temperament also promote the development of a guilt complex. The development of this complex is more likely when there is a low value for social plasticity $(\mathrm{p}<0.05)$ and high levels of emotional sensitivity $(\mathrm{p}<0.01)$. Women with these temperaments are distinguished by difficulty engaging in social contact, are restrained in their interactions, anxious, unsure of themselves, and extremely sensitive to any failure and are easily emotionally wounded.

The inclination to use the self-pity complex to compensate for a stressful state is significantly dependent on individual psychological properties. Self-pity and the belief that everyone else is hard-hearted and incapable of sympathy characterizes women with low values for personality Factor A-"reserved-caring." It is known that for such individuals the qualities of reticence, detachment, excessive severity, and skepticism in assessing other people are typical. In female inmates, these qualities are compensated for with the complexes of self-pity (the interconnection between indicators was at the level of $\mathrm{p}<0.01)$ and fear $(\mathrm{p}<0.05)$.

In turn, the fear-complex indicator is interconnected with properties of temperament. The nature of the interconnection suggests the following interpretation of the pattern: low values for social hardiness given extreme social emotional sensitivity predispose to the development of the fear complex. Evidently, under difficult conditions caused by the deprivation of freedom and forced confinement in a correctional facility, for women characterized by a narrow circle of contacts, reservedness, social passivity, and reluctance to participate in social events and who are furthermore extremely sensitive to failures in social interaction and are easily emotionally wounded, unsure of themselves, and anxious, this complex allows for the discharge of tension.

Accentuation of personality, as noted above, is also an active factor in the development of complexes. For example, the pedantic type of accentuation that characterizes rigid, passive people who experience emotion from traumatic events for a long time and at the same time are punctilious, inclined toward self-reproach, suspicious, and follow strict routines, if conditions are altered, can provoke the development of pseudo compensation in the form of the foreboding complex in female inmates $(\mathrm{p}<0.05)$.

Analysis of the nature of interconnections among the indicators we studied in male inmates suggests analogous patterns. The clearest determinants of primitive psychological defenses, as in the female sample, was, first and foremost, accentuation of character and properties of temperament, followed by personality traits. It is interesting that among the things that provoke the development of pseudo compensation in men, the way they conceptualize life, the spiritual potential of personality, and physical health stand out, things that are not seen in the structure of the female inmate sample. An especially active "trigger" is the "controllability of life" indicator for conceptual-life orientation. At first glance the interconnection appears to be a paradox: the indicator's high value for life controllability is accompanied by heightened complexes of fear and also feigned ingenuousness and gullibility $(\mathrm{p}<0.05)$. But there is a certain logic here: a person convinced that he has been endowed with the ability to control his life, who makes decisions freely and realizes them in life, is sentenced to forced incarceration and winds up in conditions where his own efforts, initiative, and right to do as he pleases, rather than being encouraged, are brutally suppressed. In addition to fear, this provokes a need to defend the ego, and one means of doing this is to demonstrate "ingenuousness": "I cannot do anything with my naivety," "I am incapable of cunning, everyone deceives me," "People take advantage of my simplicity and trusting nature," "1 always trust people and often have reason to regret this". Furthermore, actualizing the gullibility complex 
permits low mental effort. Mental passivity characterizes people who do not believe in goodness, do not expect support from others, and are not inclined toward spiritual exploration (the value for the correlation coefficient between these - two indicators was $r=-0.67$, given $\mathrm{p}<0.01$ ).

Similar patterns of interconnections among indicators are seen in the structures built on the basis of data from psychodiagnosis of the disabled, both women and men. With high reliability values $(p<0.01)$, analogous interconnections were detected between an inclination toward pseudo compensation and personality traits, accentuation of personality, and temperament properties As in the inmate sample, certain differences were seen between the structures of the female and male groups comprised of people with sight, hearing, and mobility disabilities (people confined to wheelchairs): despite large numerical values for pseudo compensation, in the "female" structure these indicators were less interconnected with other indicators (thirteen interconnections), while in the "male" structure there were significantly more-eighteen $(\mathrm{p}<0.01)$. This indicates greater functional unity of individual psychological personality properties and psychological defenses within the structure of indicators for the male sample as compared with the female one.

The data from analysis correlating indicator values diagnosed within the male and female immigrant samples also suggest interconnections between personality traits and inclinations toward using psychological defenses within the personality structures of people living under chronic stress. Just as was the case for groups of inmates and the disabled, large numbers of reliable interconnections between indicators were found in the immigrant group, and to a larger extent among men (there were fifteen interconnections for the male sample and nine for the female one).

Correlation analysis of empirical data from the diagnosis of individual psychological properties and indicators of a propensity to use pseudo compensation among subjects did not find the same patterns of interconnections between indicators in the control group as those identified in the groups living with chronic stress. There were numerous interconnections among certain indicators of individual psychological properties. But, as it turned out, these indicators essentially did not correlate with how strongly expressed complexes were among subjects, and the complexes themselves were weakly linked to one another. It can be assumed that under conditions not characterized as stressful, when a person is not required to actively implement adaptive resources, the structure of individual psychological properties does not come together as a unified complex of symptoms and indicators of psychological defenses, as is (he case for those under conditions of chronic stress.

\section{Discussions}

Russian Psychologist Bodrov V. A. mentions in his book that 'the notions of Strategy in relation with stress coping involves certain types of analyzing conditions, predispositions or signs of stress situations and person's behaviour in these conditions'.

In the author's opinion stress coping includes four groups of means: avoiding stress factors by means of regulating life and work conditions; regulating the requirements of the situation; modification of stress producing behaviour; and development of personal resources needed to overcome stress.

Foreign Psychologist H. Weber considers that psychological strategies to overcome stress include the following forms:

Cognitive or behavioural solution of the problem; 2) search of social support; 3) interpretation of the situation to the person's benefit; 4) protection and negation of the problem; 5) avoidance; 6) compassion to oneself; 7) decrease of self-esteem; and 8) emotional expression.

Cheder Williams (Williams, 2007) in his book "Speaking Picture or How to Know Your Internal Me" provides advice how to cope with stress. In order to do the exercise "Express Anxiety" Cheder Williams suggests:

To take comfortable and relaxing position;

To locate feeling of anxiety inside the body. To determine where it is settled: in the stomach, on the face, in the head or in the legs;

To take coloured pencils;

To pour out anxiety and to transfer it onto the paper with subdominant hand.

Lucia Capaccione (Capaccione, 2005) suggests drawing with both hands in order to release stress. In her opinion this exercise allows to free the mind from unnecessary sad or worrying thoughts and serves a relaxant. When feeling exhausted, upset, mentally distressed, one should try to do the exercise using both hands. 


\section{Conclusion}

The conclusions that the correlation analysis point to match contemporary psychology's views on the role of personality in adaptation. Personality is recognized as "the source and culmination of adaptive processes" (Posokhova, 2001, p. 112). S.T. Posokhova believes that to understand the essence of adaptation, it is important to keep in mind that personality regulation allows for the transformation of structural organization. Transformation implies the formation of new mental properties, the meshing of existing properties, and the manifestation of latent ones. Among the list of new properties she includes adaptedness reflecting the extent and way in which existing adaptive potential is actualized. A change to the substance of personality regulation demonstrates the high level of adaptive plasticity necessary to preserve the integrality of a personality in the face of interaction with a dynamic social environment.

The results of our research, which supports current ideas, suggest that adaptedness is an integral feature of personality, a structure formed out of the properties that enable the process of individual psychological adaptation under conditions of chronic stress. Indeed, under harsh conditions of isolation that demand adaptation, such as those experienced during incarceration, as well as the limitations associated with the partial or complete disability, or immigration, when it is impossible to change the current situation or to find other means of overcoming chronic stress, people demonstrate a certain adaptive plasticity. Preservation of the integrity of the ego and reduction of mental tension is achieved through changes made to the substance of self-regulation mechanisms in which a prominent role begins to be played by primitive psychological defenses including the pseudocompensations we investigated. People are most likely to resort to such forms of psychological defense when their freedom has been severely limited and they have been deprived of many of the opportunities fate usually offers to the socially adapted and physically healthy member of society. The research we performed permitted us to identify a number of patterns in the use of psychological defenses: it was demonstrated that their use has adaptive significance in those instances where opportunities for the personality to ensure sociopsychological adaptation while under chronic stress are clearly insufficient.

Comparative analysis of our research data showed that the way in which psychological defenses were used was determined by two things. On the one hand, it was determined by components of a personality's adaptive potential, and on the other, by the specific nature of the situation. In the second case, cognitive assessment that the situation was hopeless, beyond the person's control, as is the case during forced isolation due to incarceration, provokes actualization of the highest number of primitive psychological defenses that have "taken root" within the personality's cluster of adaptive properties. A lesser role is played by pseudocompensation that fulfills the function of psychological defense in cases of disability-related isolation. Under these circumstances, the boundaries of isolation are not so rigid and regulated, and people living in such conditions can count on some social support. The isolation experienced by immigrants is purely conditional and is primarily associated with being torn away from familiar conditions and a narrowing of the circle of interaction. Nevertheless, a personality that finds itself in difficult conditions caused by immigration experiences significant tension due to the limited circle of interactions that, as in other cases of isolation, is partially compensated for by the use of psychological defenses that are incorporated into the personality's adaptive cluster.

Comparison of results with the data of other investigators painting a psychological portrait of a personality with high adaptive potential leads to the conclusion that the level of development of properties enabling adaptation among those we diagnosed living with chronic stress was, overall, not high.

In examining the complex of symptoms being studied for gender differences it turned out that women have lower values for these properties than men, which partially explains women's greater reliance on pseudocompensation. Evidently the components of the personality structure are insufficiently adapted for this and cannot provide women with the necessary level of psychological adaptation, which forces them to resort to the use of psychological defenses. The results of this study attest to the fact that the "assortment" of compensations is better suited to their task among members of the male sample, since they more closely correspond to individual psychological properties. Correlative structures formed of indicators of individual psychological properties in the female samples feature a smaller number of interconnections and exhibit less functional unity among studied properties than seen in structures of male indicators. The degree of cohesion among these psychological properties and the nature of correlative dependencies that condition the interchangeability of and substitutability of properties is higher in male samples, which is evidence of the formation of a symptom complex of properties enabling greater adaptive resources. Judging by the nature of interconnections among studied indicators, the realization of adaptive resources among men under chronic stress occurs due to a large number of interconnections between individual psychological properties and means of psychological defense that ensure their interchangeability and substitutability. Among women experiencing chronic nervous tension, the necessary 
level of sociopsychological adaptation is supported through actualization of pseudo compensation and accentuation of individual character traits, as evidenced by the reliably higher numerical values for these indicators.

The results of this study can be used in psychological rehabilitation to apply a differentiated approach to those experiencing chronic stress associated with the partial or complete loss of freedom.

\section{References}

Bodrov, V. A. (2006). The problem of coping with stress. Psychological Journal, 122.

Bogomolov, A. M., \& Portnova, A. G. (2005). Communication intensity psychological defense with the process of self-realization in the period of active personality formation. The world of psychology, 248.

Capaccione, L. (2005). Power of a Hand. In Or how to Activate the Abilities of the Right Hemisphere of the Brain by using Left Hand (p. 336). Moscow.

Gabdreeva, G. S. (2002). Sex differences in stress tolerance. In Psychology of mental states (p. 410). Kazan.

Granovskaia, R. M. (2007). Elements of practical psychology (p. 396). St. Petersburg: Rech.

Kruzhkova, O. V. (2006). The effectiveness of the structure of psychological defenses of a teacher of primary education (Ph.D. Kazan).

Maklakov, A. G. (2001). Personal adaptive capacity: The ego mobilization and forecasting in emergency conditions. Psychological Journal, 24.

Mutlu, G. M. (2007). The Journal of Clinical Investigation. Retrieved from http://www.jci.org

Mulleneisen, B. G. (1993). The stress syndrome (p. 134). Kazan: KSU.

Popova, T. M. (2004). Coping mechanisms as a factor of protection of addictive behavior (p. 15). Tambov.

Posokhova, S. T. (2001). Personal regulation of adaptation in a changing social environment (p. 121). St. Petersburg: Siberia university.

Williams, H. B. (2007). Speaking Picture or How to know your internal Me (p. 205). Moscow: AST: ASTREL.

\section{Copyrights}

Copyright for this article is retained by the author(s), with first publication rights granted to the journal. This is an open-access article distributed under the terms and conditions of the Creative Commons Attribution license (http://creativecommons.org/licenses/by/3.0/). 